\title{
Causal Proportionality as an Ontic and Epistemic Concept
}

\author{
Jens Harbecke ${ }^{1}$ (D)
}

Received: 1 April 2019 / Accepted: 20 August 2021

(c) The Author(s) 2021

\begin{abstract}
This paper is concerned with the content of the causal proportionality constraint. It investigates two general versions of the constraint, namely "horizontal" and "vertical" proportionality. Moreover, it discusses whether proportionality is considered an ontic or an epistemic, i.e. explanatory, constraint on causation in the context of some of the most prominent theories of causation. The following main claims are defended: (1) The horizontal (HP) and the vertical version (VP) of the proportionality constraint are logically independent. (2) HP is implied by some prominent theories of causation, not by others. (3) None of the discussed popular theories of causation contradicts either HP or VP. (4) HP and VP are not ontic or epistemic principles as such; rather, whether they are ontic or epistemic depends on the theories chosen plus background assumptions about the existence of higher-level causes and their non-identity to lower-level ones.
\end{abstract}

\section{Introduction}

The notion of proportionality has attracted the interest of metaphysicians working on theories of causation in general, and the problem of mental causation in particular. However, a technical use of the term is fairly novel in philosophy. Wide-spread mention of the word has been present in the philosophical literature only since Yablo published his seminal paper on mental causation in (1992). Analyzing the relationship of supervenient and subvenient causes, he says "(...) it seems clear that faced with a choice between two candidate causes, normally the more proportional candidate is to be preferred." (277) Causes are "proportional" if they "(...) incorporate a good deal of causally important material but not too much that is causally unimportant." (274)

Jens Harbecke

jens.harbecke@uni-wh.de

http://www.jensharbecke.com

1 Department of Philosophy, Politics, and Economics, ISIC - The PPE Institute for Social and Institutional Change, Witten/Herdecke University, Alfred-Herrhausen-Str. 50, 58448 Witten, Germany 
Questions about the possibility of level-bound or macro-causation had been around for some time before Yablo's work (cf. Schumacker \& Lomax, 2004). However, the idea that proportionality is a constraint on causation seems to have been Yablo's discovery-or perhaps “invention". Yablo's paper has since had a large impact in the philosophy of mind (according to GoogleScholar, 981 citations on 22nd August 2021), and the proportionality constraint has made its career in metaphysics and the philosophy of mind accordingly (cf. among others McGrath, 1998; Shoemaker, 2003b; McLaughlin, 2007; Schröder, 2007; Crane, 2008; Woodward, 2008b; List \& Menzies, 2009; Weslake, 2013; Harbecke, 2008, 2013, 2014a; Harbecke \& Atmanspacher, 2012; Bernstein, 2014).

In this paper, I am concerned with the content, status, and application of the proportionality constraint in the context of some of the most prominent theories of causation. In particular, I investigate two general versions of proportionality, which I call "horizontal" and "vertical" proportionality, and the constraint's role in three popular classes of theories of causation.

As a very first hint, two events can be described as "vertically proportional" if they display the same degree in "graininess" and "detail". An example might be Biden's winning of the election in November 2020 and Biden's actually becoming president of the United States on 20th January 2021, both of which are macroscopic social events and comparable in their graininess and detail. This is in contrast to Biden's winning of the election in November 2020 and the vastly complex molecular event underlying Biden's actually becoming president of the United States on 20th January, which are very different in their degree of complexity and their amount of detail.

Two events can be described as "horizontally proportional" if one is "sufficient, but not redundant" with respect to the other. An example might be Suzie's throwing of the stone and the shattering of the bottle-in contrast to the state of the entire universe at the moment when Suzie throws the stone which is not horizontally proportional to the shattering of the bottle (the former contains much more than was necessary for the shattering to occur and was therefore redundant with respect to the latter).

The selected set of prominent theories of causation which I discuss in order to determine their relationships to the different versions of proportionality are interventionist theories, Humean theories, and power theories of causation. I have chosen this particular set of theories mainly because they are either among the currently most popular and widely discussed theories themselves, are or they closely related to further popular theories such as structural equation approaches or probabilistic causation. ${ }^{1}$

I also shed some light on the question whether proportionality is considered as an ontic or as an epistemic, i.e. explanatory, constraint on causation in the context

\footnotetext{
1 Process Theories, Granger Causality etc. have been left out as they seem to have fewer adherents in philosophical circles today than the ones chosen for the investigation in this article. However, their relationship to proportionality constraints would form an interesting research topic for future research.
} 
of these theories. Both positions can be found in the mental causation literature, and their defense has been a matter of controversy.

As an answer to these questions I will defend the following main claims ${ }^{2}$

1. The horizontal (HP) and the vertical version (VP) of the proportionality constraint are logically independent; they do not entail each other or the other's negation.

2. HP is implied by some of the currently most popular theories of causation.

3. None of the currently most popular theories of causation contradicts either HP or VP. They could embrace HP and VP as further constraints, without rendering the underlying theory of causation incoherent.

4. HP and VP are not ontic or epistemic principles as such; rather, whether they are ontic or epistemic depends on the theories chosen plus background assumptions about the existence of higher-level causes and their non-identity to lower-level ones.

The paper is structured as follows. In a first step (Sect. 2), I review some contributions in the philosophical literature that explicitly discuss, and in some cases adopt, Yablo's proportionality constraint. Some of these will be characterized as interpreting the constraint as an ontic, some as an epistemic concept. Afterwards (Sect. 3), I offer a preliminary definition of the notion and I distinguish HP and VP more specifically. In a next step, I clarify the status of the proportionality constraint from the perspective of three popular but fundamentally different theories of causation, namely interventionist, Humean, and power theories of causation (Sects. 4-6). Section 7 summarizes the results and points to some puzzles in the context of the proportionality constraint that will have to be left for future research.

\section{Proportionality in the Philosophy of Mind}

As mentioned in the previous section, Yablo (1992) believed that from the set $\mathcal{C}:=\left\{C_{1}, C_{2}, \ldots, C_{n}\right\}$ of all sufficient causes of a given effect $E$, usually only a subset is, or should be, considered the actual or genuine cause(s), namely the cause(s) that is (are) proportional to $E$. Sufficient causes are proportional to a given effect if they "(...) incorporate a good deal of causally important material but not too much that is causally unimportant." (274) Yablo himself explicates this idea on the basis of four conditions specifying a specific kind of counterfactual dependency of an effect

\footnotetext{
2 The reader may wonder why my list of claims does not include an answer to the normative question whether (and if so why) we should adopt proportionality as a constraint, and whether we should think of it as an ontic or an epistemic constraint. Without doubt, these are interesting questions. However, given what I demonstrate in the following sections, one should expect the answer to depend heavily on the theory of causation that one accepts, as well as on one's take on the existence of non-fundamental facts or events. Both commitments require detailed arguments that go far beyond the aims of the present paper. Hence, at least for the time being, I encourage the reader to make her own conclusions with respect to these further normative questions, and I prefer not to take a strong stance myself.
} 
$E$ on candidate causes: A cause $C$ is proportional with respect to an effect $E$, if and only if, $E$ is contingent upon $C^{3}, C$ is adequate for $E, C$ is required for $E$, and $C$ is enough for $E$. Contingency amounts to a kind of counterfactual dependence of $E$ on $C$. Adequacy implies a counterfactual sufficiency of $C$ for $E$. Requiredness demands that any impoverishment in detail of $C$ would have led to the non-occurrence of $E$. Finally, enoughness declares that the genuine cause $C$ should mark the upper bound of all events that satisfy requiredness with respect to $E$.

The earliest explicit response to this idea is found in a paper by Green and Vervaeke (1996), who agree with Yablo that “(...) given different effects of the same precipitating event, different aspects of the precipitator will move to the fore as more plausible candidates for the title of "cause'." (205) Critical responses have been elaborated, among others, by McGrath (1998) and Gillett and Rives (2005). Whereas McGrath believes that " $(. .$.$) the notions of proportionality and of properties as deter-$ minables cannot be used, jointly, to solve the problems raised by BELOW [also called the "exclusion problem"] and WITHIN" $(170)^{4}$, Gillett and Rives simply deny that there are such determinable properties that could be subject to proportionality.

A more recent version of the proportionality constraint has been offered by Shoemaker (2003b), who develops a theory of mental causation on the basis of his power theory of properties. About the alleged competition of two properties $P_{1}$ and $P_{2}$, where $P_{2}$ contains a subset of the powers of $P_{1}$, he says:

Yablo's notion of proportionality can be applied here. Where the only causal features of property $P_{1}$ that play a role in producing an effect are ones that belong to property $P_{2}$, of which $P_{1}$ is a determinate or realizer property, there seems a good sense in which considerations of proportionality favor the instantiation of $P_{2}$ over the instantiation of $P_{1}$ as a cause of the effect. (Shoemaker, 2003b, 436)

Whereas Shoemaker believes that proportionality is an ontic feature of causation since, in his view, properties are objective features of the real world, Thomas Bontly points out that it is mostly a pragmatic phenomenon within our use of causal language. Consequently, he denies that non-reductive physicalism can claim support from the constraint: “(...) proportionality is not in fact a constraint on the causal relation but rather a pragmatic feature of our use of causal language, derived from general principles of language use. If so, it turns out that proportionality has little if anything to do with the nature of causation itself and cannot save nonreductive materialism from the exclusion problem." (Bontly, 2005, 332)

\footnotetext{
3 Yablo defines the contingency of an event $E$ on an event $C$ as counterfactual dependence: If $C$ had not occurred, then $E$ would not have occurred either" (cf. Yablo, 1992, 274). Concerning the evaluation of such conditionals, Yablo follows Stalnaker and Lewis (cf. Yablo, 1992, fn. 56).

4 McGrath summarizes his argument as follows: "If Yablo is to adequately answer WITHIN, he must give us an acceptable account of determination that rules out disjunctive properties as determinates of their disjuncts. If he is to adequately answer BELOW, he needs not only, (i), to provide such an account of determination, but (ii), to tell us what pain is if it is not a disjunctive property the disjuncts of which are its underlying brain states. I have given reasons for doubting that (i) and (ii) can be jointly discharged in the context of Yablo's account." (McGrath, 1998, 173)
} 
Brian McLaughlin has similar inclinations as Bontly towards an epistemic, pragmatic, or explanatory interpretation of proportionality. As he says, "[f]or the record, I myself think that rather than a constraint on causation, proportionality is a pragmatic constraint on explanation. Too much causal detail or too little causal detail makes for a poor or misleading explanation in a context." (McLaughlin, 2007, 15/16)

Since the work of Shoemaker, Bontly, and McLaughlin, reference to proportionality has become frequent in the philosophy of mind-both in an appreciative and a critical manner. Generally appreciative examples are Schröder (2007), Crane (2008), Woodward (2008b), and List and Menzies (2009), Weslake (2013), Harbecke (2013, 2014a), and Harbecke and Atmanspacher (2012). Another critical view has recently been voiced by McDonnell (2017), who argues that the proportionality principle cannot be a strong constraint on causation as it violates much of our practice in identifying causal relations.

In short, over the last years the notion of proportionality has received much attention in metaphysics and the philosophy of mind. At the same time, a disagreement re-occurs about the status of proportionality as an ontic or epistemic concept. This disagreement forms the background for the following sections.

\section{Two Kinds of Proportionality}

Apart from the ontic/epistemic disagreement mentioned at the end of Sect. 2, the debates on mental causation and the nature of causation have dealt with two very different kinds of causal proportionality, which could be characterized as "vertical" and "horizontal" proportionality. ${ }^{5}$ As a first and non-technical approach", these two notions can be characterized as follows:

Vertical Proportionality (VP): An event $\phi$ is vertically proportional to an event $\psi$ if, and only if, $\phi$ and $\psi$ display the same degree in "graininess" and "detail" within a supervenience hierarchy, i.e. a hierarchy that involves ordered sets of events all of which occur in the same place and time, but where every event is sufficient for all events located at a higher position within the hierarchy. ${ }^{7}$

Horizontal Proportionality (HP): An event $\phi$ is horizontally proportional to an event $\psi$ if, and only if, $\phi$ is sufficient, but not redundant with respect to $\psi$, where

\footnotetext{
5 Contributions discussing proportionality in the context of mental causation, such as McGrath (1998), McLaughlin (2007), Crane (2008), Woodward (2008b), List and Menzies (2009), Weslake (2013), and Bernstein (2014) mostly focus on vertical proportionality. Others mainly concerned with the nature of causation have discussed versions of horizontal proportionality sometimes implicitly in the context of minimality conditions. These include Mackie (1974), Graßhoff and May (2001), May (1999), Baumgartner and Graßhoff (2004), and Baumgartner (2008). In other contributions, both notions seem to be present, such as possibly Shoemaker (2003b), and Schröder (2007).

6 For some recent attempts to define proportionality in a technical way, see Pocheville et al. (2017) and Gebharter and Eronen (2021).

7 Note that a "supervenience hierarchy" can in principle comprise events with the same degree of graininess level only, as any set of events trivially supervenenes on itself.
} 


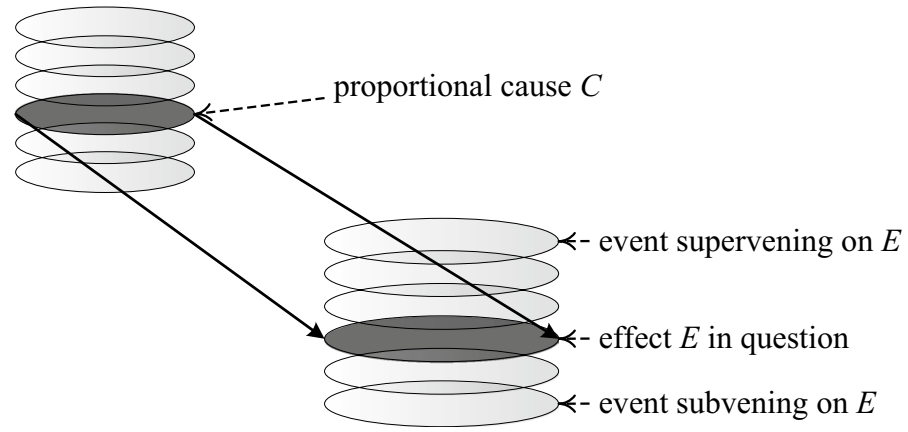

Fig. 1 Vertical Proportionality

"redundancy" compares non-identical events with a similar degree of graininess which may or may not overlap in at least one subevent.

Characterizations VP and HP are intended as merely preliminary definitions. Among other things, they make use of a "similarity in graininess" relation in a supervenience hierarchy without offering a further definition of this notion. Moreover, depending on the theory of causation presupposed, VP and HP will have to receive a different formulation. Nevertheless, the two definitions may help to approach the two kinds of proportionality in a heuristic way.

Some features of VP and HP should be mentioned here already. First, note that neither VP nor HP binds proportionality to a causal relation. An event $C$ might satisfy VP with respect to another event $E$ without being causally connected to that event. For instance, the event "the audience's applause at the Zurich Opera" may display a similar degree of graininess as the simultaneous event "the audience's applause at the Berlin opera", because the two events are both meso-scale entities that involve collections of people. But the applause in Zurich may not be causally connected in any way to the applause in Berlin. In a similar way, an event $C$ can satisfy HP with respect to an event $E$ without being causally related to $E$. Assume $E_{1}$ and $E_{2}$ are both effects of a single common cause $C C$. Assume further that $C C, E_{1}$ and $E_{2}$ are all part of a deterministic causal system such that whenever $E_{1}$ occurs, $E_{2}$ occurs (because in this case $C C$ also occurs) and that whenever $E_{1}$ does not occur, $E_{2}$ does not occur too (because in this case $C C$ does not occur). $E_{1}$ is sufficient, but not redundant with respect to $E_{2}$ in this scenario, but $E_{1}$ is neither a cause nor an effect of $E_{2}{ }^{8}$

The way vertical proportionality acts in causal situations is illustrated by Fig. 1. The two layered cylinders represent sequences of events, all of which fully overlap and which are ordered by the relation of supervenience. The arrows represent actual, or proportional, causal relationships. Consider, for instance, the following three events that (presumably) fully overlap and are ordered by supervenience: $C_{1}^{\prime}=$ Otto

\footnotetext{
${ }^{8}$ I thank an anonymous referee for bringing this point about horizontal proportionality to my attention.
} 


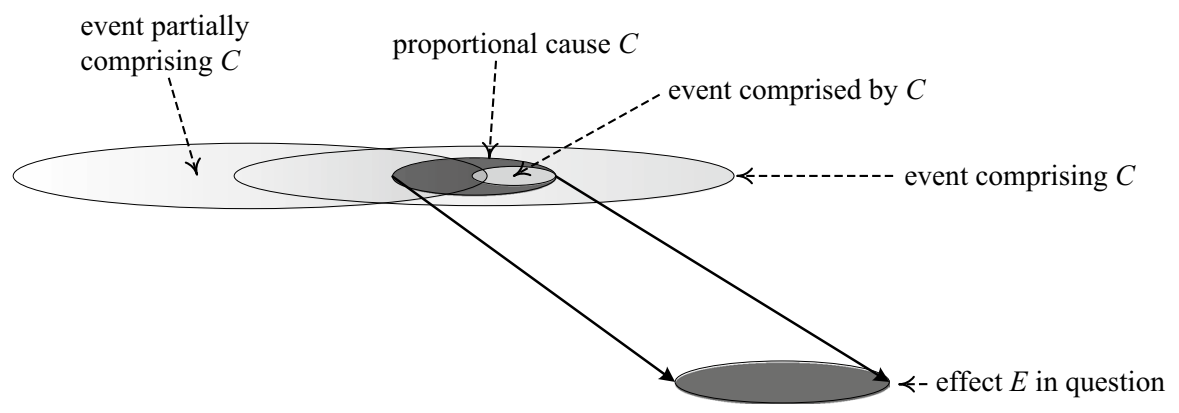

Fig. 2 Horizontal Proportionality

cycling to the grocery store, $C_{2}^{\prime}=$ Otto cycling to the grocery store fast, $C_{3}^{\prime}=$ Otto cycling to the grocery store fast and furiously $\left(C_{1}^{\prime}\right.$ supervenes on $C_{2}^{\prime}$ supervenes on $C_{3}^{\prime}$ ). For such vertical sequences of events all of which are sufficient (and non-redundant) for a certain effect (perhaps $E^{\prime}=$ the truck driver shouting at Otto for his way of driving on the road), VP is meant to attain a match between the specificity of the cause to the effect. For instance, it might turn out that $C_{3}^{\prime}=$ Otto cycling to the grocery store fast and furiously was proportional to the truck driver's shouting and, hence, the actual cause (Otto's mere cycling would not have sufficed for the driver to shout at him).

Or consider the following three events which may satisfy the same conditions of supervenience and overlap despite not being conceptually connected: $C_{1}^{\prime \prime}=$ the activity of a complex neural cell network in my central brain, $C_{2}^{\prime \prime}=$ the activation of my amygdala, $C_{3}^{\prime \prime}=$ my mental state of sadness in response to disturbing news about the Syrian civil war (ex hypothesis: $C_{3}^{\prime \prime}$ supervenes on $C_{2}^{\prime \prime}$ supervenes on $C_{1}^{\prime \prime}$ ). And take the effect in question to be $E^{\prime \prime}=$ my switching to a website with fun information about sunny holiday destinations. Then $C_{3}^{\prime \prime}$, or possibly $C_{2}^{\prime \prime}$, might turn out to be proportional to $E^{\prime \prime}$ under the assumption that the neural network activity was by far too specific to be located on the same graininess level as $E^{\prime \prime}$. Hence, according to VP, $C_{3}^{\prime \prime}$ and $C_{2}^{\prime \prime}$ are more suitable candidates for causing $E^{\prime \prime}$ than is $C_{1}^{\prime \prime}$.

Horizontal proportionality approaches the matter from a different angle. The idea is illustrated by Fig. 2. The comparison made by $\mathbf{H P}$ is one between two or more sufficient causes. However, in this case the non-proportional events are redundantthey occupy "too much" spacetime, or contain too many irrelevant subevents-with respect to the effect. The notion of a subevent is not metaphysically innocent here, of course. That many events contain further events as parts or subevents seems hard to deny nonetheless. WWII contains the subevent of the bombing of the Warsaw Ghetto, Cindy's and John's wedding contains as a subevent the exchanging of the rings, Usain Bolt's world record run in Berlin contains as a subevent him crossing the victory line etc. In some cases, not all of the subevents of an event are causally relevant for a given effect. It is such redundant events that $\mathbf{H P}$ is supposed to declare as causally relevant for, but as not actually causing, the effect.

An example might help to illustrate the idea. If $C_{1}^{*}$ is the entire macroscopic state of the universe at $t_{1}, C_{2}^{*}$ is the movement of my hand at $t_{1}$ (such that $C_{2}^{*}$ is contained 
or included in $C_{1}^{*}$ ), and $E^{*}$ is the tipping over of my wine glass at $t_{2}$, then it seems that $C_{2}^{*}$ is, and $C_{1}^{*}$ is not, horizontally proportional to $E^{*} . C_{1}^{*}$ may contain such things as Melania Trump's combing of her hair, Bruno Mars giving a concert in Tokyo etc.- - two subevents of the total state of the universe at $t_{1}$ that, as far as we can tell, are far too remote to have an impact on my glass at $t_{2}$. Consequently, $C_{1}^{*}$ is not wellcalibrated with respect to $E^{*}$. It contains massive amounts of irrelevant surplus and, according to HP, is therefore disqualified as a causes of $E^{*}$.

Whether HP is a justified or adequate ingredient in our theories of causation is a different question that cannot be answered in depth here. However, to offer a hint: A strong reason for believing that redundancy as targeted by $\mathbf{H P}$ is a problem for causation is that it violates a basic and widely accepted constraint on ontology and science. The constraint is often dubbed "Ockham's razor" alluding to the 14th century scholar William of Ockham who famously demanded: Numquam ponenda est pluralitas sine necessitate ["Plurality must never be posited without necessity"]. The fundamental idea has received various formulations in many different philosophical and scientific contexts (cf. Baker, 2016). The principle has sometimes been justified on a priori grounds. One such defense claims that redundancy violates our aesthetic sense and our standards of rationality. Naturalistic justifications have cited as empirical evidence the patterns of acceptance and rejection of competing theories by working scientists (cf. Baker 2016, sections 3-4.)

Given that these justifications are roughly valid, it would be peculiar if parsimony constrained our ontological and scientific attitudes, but turned out to be inapplicable to our theories of causation. ${ }^{9}$ Or, in other words, if the justifications of the parsimony principle are convincing in other fields, we probably want to let them guide us in our attempt to distinguish causes from non-causes as well. Otherwise, the number of causes of each everyday event would simply explode in an intractable fashion.

The two conditions of VP and HP, both under an ontic and an epistemic reading, are logically independent. A cause can in principle satisfy both, only one, or none of the two conditions. To offer a rigorous proof for this claim requires a formal explication of VP and HP. Depending on the theory of causation presupposed, the formalizations will be different in each case. A more accessible and shorter way to support the independence claim is again by an example.

Consider the large mesoscopical event $C_{2}^{*+}$, which may be the bodily movements of all party guests at $t_{1}$ including $C_{2}^{*}=$ the movement of my hand at $t_{1}$. Since both $C_{2}^{*+}$ and $E^{*}=$ the tipping over of my wine glass at $t_{2}$ are mesoscale events, and since $C_{2}^{*+}$ had something to do with the occurrence of $E^{*}$ (precisely because it contained $\left.C_{2}^{*}\right), C_{2}^{*+}$ can be expected to satisfy VP with respect to $E^{*}$, at least relative to $C_{2}^{*+^{\prime}}$, which is the total microphysical event underlying $C_{2}^{*+}$. The reason is that, on any account, $C_{2}^{*+^{\prime}}$ is on a much lower level than $E^{*}$. At the same time, $C_{2}^{*+}$

\footnotetext{
9 It should be noted that some authors have expressed doubts concerning the analogy of science and metaphysics and the applicability of simplicity constraints to both (cf. Huemer, 2009; Kriegel, 2013; Willard, 2014). Due to limits of space, I cannot enter this debate in much depth here, but tend to believe Brenner (2017) when he says that if you accept the idea that simplicity is truth-conducive in science, it would be objectionably arbitrary to reject the idea that simplicity is truth-conducive in metaphysics.
} 
can be expected to fail HP with respect to $E^{*}$, at least if $C_{2}^{*}$ is taken into account. That is because $C_{2}^{*+}$ contains much irrelevant stuff including Jenny waving Dave to come over to the bar. $C_{2}^{*}$, in contrast, can be expected to fail VP with respect to $E^{*^{\prime}}$, which is the total microscopic state underlying $E^{*}$. That is because, on any account, $C_{2}^{*}$ is on a much higher level than the microscopic event $E^{*^{\prime}}$. However, $C_{2}^{*}$ may still satisfy HP with respect to $E^{*^{\prime}}$, because a much smaller region than the one covered by $C_{2}^{*}$ might not have been enough to secure the occurrence of $E^{*^{\prime}}$.

A better candidate for the satisfaction of VP with respect to $E^{*^{\prime}}$ might have been $C_{2}^{*^{\prime}}=$ the microphysical and microscopic state underlying $C_{2}^{*}$. However, the event $C_{2}^{*+^{\prime}}=$ the microphysical and microscopic state underlying $C_{2}^{*+}$ should be expected to satisfy neither HP nor VP with respect to $E^{*}$ (= the tipping over of my wine glass at $\left.t_{2}\right)$. Or in short, $\operatorname{sat}\left(V P \mid\left\langle C_{2}^{*}, E^{*}\right\rangle\right)=1$ and $\operatorname{sat}\left(H P \mid\left\langle C_{2}^{*}, E^{*}\right\rangle\right)=1$; $\operatorname{sat}\left(V P \mid\left\langle C_{2}^{*+}, E^{*}\right\rangle\right)=1$ and $\operatorname{sat}\left(H P \mid\left\langle C_{2}^{*+}, E^{*}\right\rangle\right)=0 ; \quad \operatorname{sat}\left(V P \mid\left\langle C_{2}^{*}, E^{*^{\prime}}\right\rangle\right)=0 \quad$ and $\operatorname{sat}\left(H P \mid\left\langle C_{2}^{*}, E^{*^{\prime}}\right\rangle\right)=1 ; \operatorname{sat}\left(V P \mid\left\langle C_{2}^{*+^{\prime}}, E^{*}\right\rangle\right)=0$ and $\operatorname{sat}\left(H P \mid\left\langle C_{2}^{*+^{+}}, E^{*}\right\rangle\right)=0$. This exhausts all logical possibilities.

How are VP and HP related to Yablo's constraints? As mentioned above, Yablo defines causal proportionality over four conditions: A cause $C$ is proportional with respect to an effect $E$, if and only if, $E$ is contingent upon $C, C$ is adequate for $E, C$ is required for $E$, and $C$ is enough for $E$. Contingency amounts to a version of counterfactual dependence of $E$ on $C$. Adequacy implies a counterfactual sufficiency of $C$ for $E$. Requiredness rules that less detail of $C$ would have led to the non-occurrence of $E$, whilst enoughness declares that the genuine cause $C$ should mark the upper bound of all events that satisfy requiredness with respect to $E$. Since counterfactual dependency and sufficiency are not sensitive to detail but simply to the occurrence or non-occurrence of events, one interpretation is that contingency and adequacy jointly embody HP. They are designed to a select an event that is "just of the right size" to be the cause. In contrast, requiredness and enoughness are explicitly designed to isolate an event that has "just the right amount of detail" to be the cause. Hence, the two latter conditions can be interpreted as jointly embodying VP.

In this sense, the application of proportionality constraints such as the one developed by Yablo or the one re-constructed by Harbecke (2014a; without Yablo's essentialist metaphysics) can be thought of as criteria that "zoom in" on a proportional cause relative to a given effect. Partially and wholly irrelevant events are kicked out from the elected class of candidate causes, and within the supervenience hierarchy only such events pass the test that are akin to the effect in detail and specificity. Figure 3 is intended to illustrate this process of "zooming in" on the cause.

The following Sects. 4-6 aim to clarify what the relationship is between VP, HP, and some of the most prominent theories of causation. The aim is to show that HP is implied at least by some theories of causation, but not by others, and that some theories of causation accept versions of HP and VP as additional constraints. Moreover, it is argued that none of the currently most popular theories of causation contradicts either HP or VP as such. They could embrace HP and VP as further constraints, without rendering the underlying theory of causation incoherent. An inconsistency is attained with the help of additional assumptions only. 


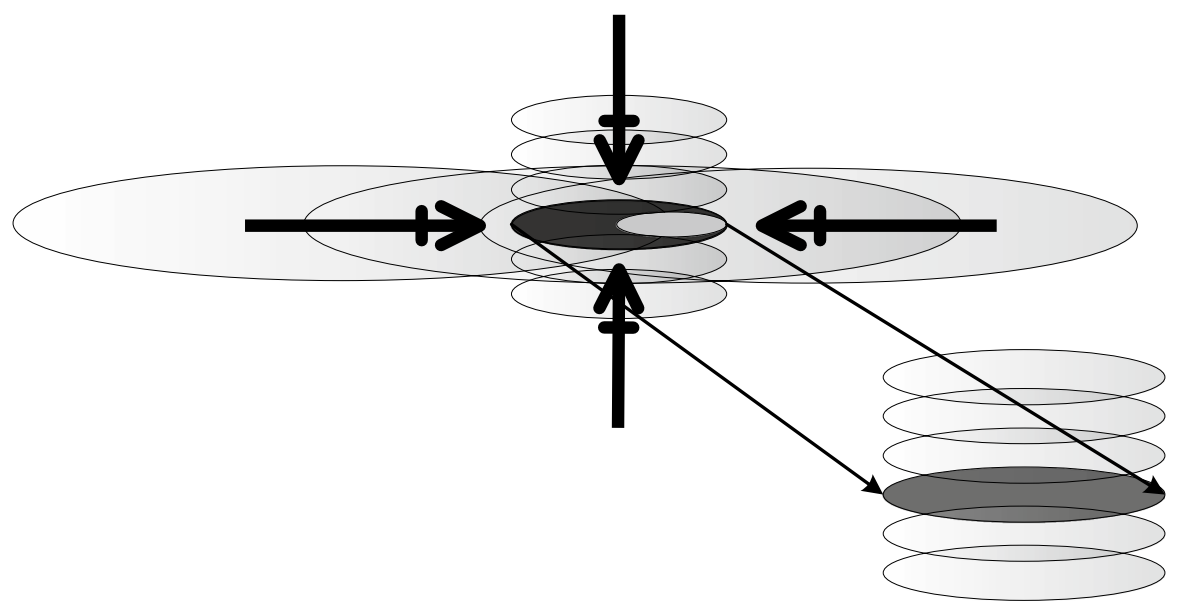

Fig. 3 "Zooming in" on a cause relative to an effect

\section{Interventionist Theories}

The basic idea of interventionism is that a variable $X$ is a cause of a variable $Y$ if, and only if, there is an ideal intervention on $X$ that brings about a change in $Y$ without $I$ being connected to $Y$ through paths not involving $X$. Woodward offers the following definition for a (type-level) direct cause, a contributing cause, and an ideal intervention:

(M) A necessary and sufficient condition for $X$ to be a (type-level) direct cause of $Y$ with respect to a variable set $\mathbf{V}$ is that there be a possible intervention on $X$ that will change $Y$ or the probability distribution of $Y$ when one holds fixed at some value all other variables $Z_{i}$ in $\mathbf{V}$. A necessary and sufficient condition for $X$ to be a (type-level) contributing cause of $Y$ with respect to variable set $\mathbf{V}$ is that (i) there be a directed path from $X$ to $Y$ such that each link in this path is a direct causal relationship (...), and that (ii) there be some intervention on $X$ that will change $Y$ when all other variables in $\mathbf{V}$ that are not on this path are fixed at some value. (Woodward, 2003, 59)

(IV) $I$ is an intervention variable for $X$ with respect to $Y$ iff "[(i)] $I$ causes $X$; [(ii)] $I$ acts as a switch for all the other variables that cause $X(. .$.$) ; [(iii)] any directed$ path from $I$ to $Y$ goes through $X(\ldots)$; [(iv)] $I$ is (statistically) independent of any variable $Z$ that causes $Y$ and that is on a directed path that does not go through X." (op. cit., 98)

In Woodward's original contributions, the notion of causal proportionality plays no explicit role. It was only when Woodward, and along with him several other authors (cf. List and Menzies, 2009; Shapiro, 2010; Shapiro \& Sober, 2007; Raatikainen, 2010; Weslake, 2009) began to argue that interventionism provides a stable basis for a non-reductive theory of mental causation and higher-level 
causation in general that proportionality entered the picture. In the scenario discussed by Woodward (2008b, 2015) and classically presented by Kim (2003), the question asked is whether a mental event or variable $M_{1}$ can cause a mental variable $M_{2}$ if $M_{1}$ has (by hypothesis) a physical supervenience base $P_{1}$ and $M_{2}$ has a physical supervenience base $P_{2}$ such that $P_{1}$ is the cause of $P_{2}$. It seems plausible that under these conditions at least some interventions on $M_{1}$ (which induce immediate changes in $P_{1}$ ) have an effect on $M_{2}$ (because, by hypothesis, $P_{1}$ influences $P_{2}$, and at least some changes in $P_{2}$ are correlated with changes in $M_{2}$ ).

Moreover, it may also be the case that some interventions on $P_{1}$ sometimes lead to changes in $M_{2}$, but in a less reliable way. Since, in Woodward's view, reliability, or stability, are additional criteria that causal relations satisfy, there are reasons to believe that the relation $M_{1} \rightarrow M_{2}$ is a causal relation in a more substantial sense than the relation $P_{1} \rightarrow M_{2}$. Woodward himself observed the analogy between the stability criterion and Yablo's proportionality constraint:

I have argued (...) that even if both $M_{1}$ and $P_{1}$ cause $M_{2}$, there may be important differences between the $M_{1} \rightarrow M_{2}$ causal relationship and the $P_{1} \rightarrow M_{2}$ causal relationship: depending on the details of the case, the causal relationship between $M_{1}$ and $M_{2}$ may be more stable or better satisfy certain other desiderata, such as "proportionality" than the causal relationship between $P_{1}$ and $M_{2}$. (Woodward, 2015, 304)

Woodward does not make explicit what kind of proportionality he has in mind here: whether it is VP or HP. However, since his concern in the present context are "macroscopic properties that supervene on (or are 'realized by') other (typically microphysical) properties but that are not identical with those realizing properties" (Woodward, 2015, 303), it becomes clear that Woodward does not have in mind HP. HP is applied to single out a cause from a set of properties or events that display the same degree of graininess which may or may not overlap (cf. Sect. 3). In other words, if one out of $M_{1}$ and $P_{1}$ is more proportional to $M_{2}$ than the other, it must be in the sense of VP.

Secondly, it is not entirely clear from this passage whether Woodward considers proportionality to be an additional constraint on causation, or whether he believes that VP is already implied by his theory. In an earlier paper, Woodward's tendency seems have been towards the second option:

From an interventionist perspective, there are circumstances in which higher level causal claims may be more satisfactory (roughly when they figure in answers to a wider range of what-if-things-had-been-different questions or perhaps just provide answers to such questions that a lower level account does not provide) and also circumstances in which lower level claims will be more satisfactory (roughly when they involve relationships that are more stable or variables that are more well-defined for the purposes of intervention). (Woodward, 2008a, 183) 
Under this interpretation, the criteria offered by interventionism already imply VP, and VP is not an additional constraint on interventionist causation. ${ }^{10}$

In contrast, in the most recent contribution on the topic, Woodward describes proportionality as one of the criteria for "...choosing among explanations and causal representations that we are able to exhibit or formulate" $(2021,5)$. He offers the following new formulation of proportionality:

Woodward Proportionality $\left(\mathbf{P}^{*}\right)$ : Suppose we are considering several different causal claims/explanations formulated in terms of different variables and representing different claims about patterns of dependency relations involving some target effect or explanandum $E$ and where all of these satisfy some minimal interventionist condition [...]. Then, other things being equal, we should prefer those causal claims/explanations that more fully represent or exhibit those patterns of dependence that hold with respect to $E$. (Woodward, 2021, 11)

This suggests that Woodward considers proportionality as an additional constraint on causation that is not already implied by his original theory. At the same time, in his $(2015,305, \mathrm{fn} .19)$, Woodward emphasizes: "I do not regard proportionality as a necessary condition for causation." Hence, whilst Woodward considers VP to play a prominent role in his theory, the status of this constraint relative to the central notions of the theory remains somewhat unclear.

What about HP? Does it play any interesting role in the context of interventionist theories of causation? The term as such clearly does not appear in Woodward's writings. However, the problem of redundancy does appear in the debate at various places, namely as part of the problem of variable choice. Intuitively, if $X$ represents the state of the whole universe preceding the tipping over of my wine classe $Y$ (cf. Sect. 3), then $X \rightarrow Y$ will probably satisfy (M). However, $X$ will be grossly redundant (non-proportional) with respect to $Y$. In (2016, Sect. 11) Woodward actually discusses a version of the problem of variable choice, and he assigns criteria such as specificity and sparseness as relevant for such choices:

Causes that are non-specific figure in non-sparse causal representations in the sense that variables in such representations are connected to many other variables. Sometimes representations involving variables figuring in non-specific causal relationships can be replaced with representations involving new variables standing in more specific causal relationships (Woodward, 2016, 1070)

\footnotetext{
${ }^{10}$ It should be noted here that Woodward's theory of mental causation has been criticized by Baumgartner $(2009,2010,2013)$ as well as Gebharter (2017) for technical reasons. In brief, the problem is that there can be no intervention on $M_{1}$ that is not an intervention on $P_{1}$. Since this violates condition (ii) of Woodward's definition (M), interventionism implies that $M_{1}$ can never be a cause. In my view, Baumgartner is right on this point. However, once Woodward accepts that supervenient variables are excluded from condition (M)(ii), if he accepts his stability etc. criteria, and if he allows higher-level variables into his variable sets, his definitions do yield the result that higher-level causal relations exist. The challenge for Woodward is, of course, to argue for this modification of his account on independent grounds.
} 
For instance, one may sometimes seek "to replace a representation according to which many different, disparate factors causally influence disease $D$ with a representation in which a single factor E is the proximate cause of D." (Woodward, 2016, 1070)

The demanded strategy of replacement of an non-sparse variable by a sparse one realizes the core idea of HP, namely that causes are generally non-redundant. Sparseness or HP does not enter Woodward's definition of causation. Nevertheless, by recommending it as a choice criterion, Woodward seems to make a distinction between "better" and "worse" variables and, thereby "better" and "worse" causal models. Hence, the best causal models seem to observe this criterion in Woodward's view.

And so it seems that, whilst VP and HP are not strictly implied by the most recent versions of Woodward's theory, they (or very similar notions) do play a prominent role in his interventionist theory of causation. It remains to be asked whether the two criteria should be considered as epistemic or ontic concepts in Woodward's framework. For answering this question, it is important to note that the interventionist theory of causation is primarily a theory about causal inference and causal explanation. It is, first and foremost, epistemic in this sense. It is meant to establish our best and perhaps pragmatically most useful causal hypotheses. This interpretation is supported by the fact that Woodward defends a non-reductive analysis of causation (Woodward, 2003, 20-22). His technical approach merely aims to clarify the interconnection of certain concepts, and not to provide an analysis.

Secondly, the relevant relata of the causal relation as defined by (M) and (IV) are variables. In his definition (M) (Woodward, 2003, 59), Woodward says “...for $X$ to be a (type-level) direct cause of $Y . .$. . He does not use a formulation such as "the causal claim that $X$ causes $Y$ is true if, and only if...". Nevertheless, $X$ and $Y$ are supposed to be variables, not events (even though, of course, the former is intended to represent the latter). If variables are considered to be linguistic or epistemic tools primarily, it will seem like a category mistake to characterize variables-and the relationships holding between them-as ontic. On the other hand, variables can be understood as direct representations for abstract properties as well. ${ }^{11}$ It may be more convenient to talk about variables than about abstract properties, but the direct reference gives variables an ontic taste. Nonetheless, even such ontically hued variables serve epistemic functions first and foremost, and the relations holding among them are primarily epistemic at least in this sense.

In conclusion, it seems that VP and HP are neither strictly implied by, nor explicitly contained in, the interventionist theory of causation. However, Woodward does recognize the importance of these criteria at least in some interventionist inferences, and they are consistent with his theory. Moreover, in his view, the two criteria work on a different level and are independent from another. In this sense, a cause that is more proportional in Woodward's view can be either vertically, horizontally, or vertically as well as horizontally more proportional. Woodward grants that both criteria are used in search for relatively better causal models in the framework of

11 I would like to thank an anonymous referee for pointing this out to me! 
interventionism. And since interventionism deals with causation mostly on the epistemic side, VP and HP as well become (primarily) epistemic concepts.

\section{Humean Theories}

David Hume famously defined a cause as "...an object followed by another, and where all the objects, similar to the first, are followed by objects similar to the second. Or, in other words, where, if the first object had not been, the second never had existed." (1748, Sect. 7) Hume's interpreters have seen two related but different theories of causation in this quote. According to the first one, causation consists of perceived regularities; and wherever there is a regularity observed (that fails to be falsified), there is a reason to infer the presence of a causal relationship. According to the second one, causation has essentially a modal dimension: whether an event is a cause at the actual world depends on its (non-)correlation with its potential effects in non-actual scenarios. Both theories have in common that they do not believe in necessary connections. Whether $A$ causes $B$ purely depends on the contingent distribution of properties and facts.

Modern regularity theorists such as Mackie (1974), May, Graßhoff, and Baumgartner (cf. Graßhoff \& May, 2001; May, 1999; Baumgartner \& Graßhoff, 2004; Baumgartner, 2008) have used this fundamental idea to develop more technically sophisticated theories of causation. Mackie focused on an INUS condition which answers to the problem of monotony for first order universal conditionals.

Let ' $A$ ' stand for the INUS condition-in our example, the occurrence of a short-circuit at that place-and let ' $\mathrm{B}$ ' and ' $\neg \mathrm{C}$ ' (that is, 'not- $\mathrm{C}$ ', or the absence of $\mathrm{C}$ ) stand for the other conditions, positive and negative, which were needed along with A to form a sufficient condition of the fire-in our example, B might be the presence of inflammable material, $\neg \mathrm{C}$ the absence of a suitably placed sprinkler. Then the conjunction ' $\mathrm{AB} \neg \mathrm{C}$ ' represents a sufficient condition of the fire, and one that contains no redundant factors; that is, $\mathrm{AB} \neg \mathrm{C}$ is a minimal sufficient condition for the fire. (Mackie, 1974, 246)

Graßhoff and May eventually managed to solve the additional "Manchester Hooters Problem"12 that still bothered Mackie by the introduction of a minimal theory (cf. Graßhoff \& May, 2001, 16; cf. also Baumgartner, 2008, 340). A further extension of this theory has recently been developed by Baumgartner and Falk (2019).

The question is whether the notion of causal proportionality plays any role for these modern regularity theories. In order to see the connection, it is important to

\footnotetext{
12 The problems concerns non-causal regularities. For example, assume that when the hooters go off in London, the workers in London go home. Similarly, when the hooters go off in Manchester, the workers in Manchester go home. Hence, when all potential other causes of the London workers going home can be excluded, it is possible to infer that, if the Manchester hooters go off, the London workers go home. However, this regularity should not be considered as representing a causal relationship. Hence, the regularity theorist has to include some additional constraint into her theory of causation for that theory to count as adequate.
} 
Fig. 4 Proportionality for regularities. Dotted arrows represent causal relationships; full arrows represent supervenience relationships

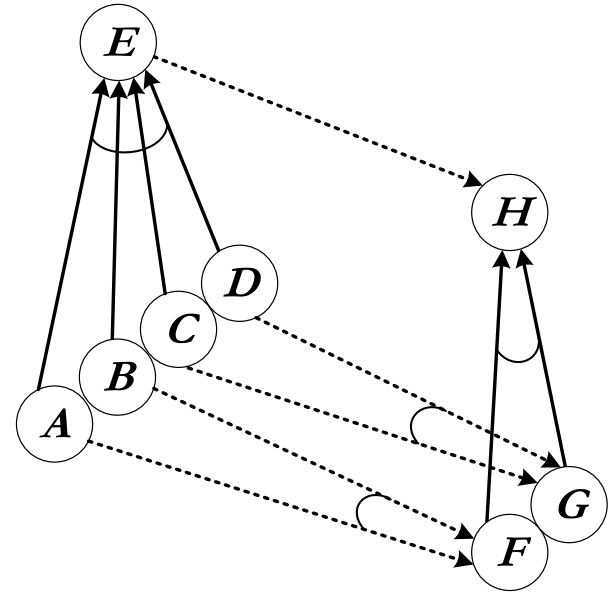

distinguish between Mackie's causes as INUS conditions, and a complete cause, which is a conjunction of INUS conditions. It makes little sense to search for non-redundancy in individual INUS condition given the fact that, by definition, an INUS condition is insufficient for the occurrence of an effect. However, the non-redundancy or minimization constraints mentioned by Mackie $(1974,76)$ and introduced by May, Graßhoff, and Baumgartner, which apply to complete causes, reflect HP: a factor $F$ is causally relevant for a factor $G$ only if it stays within a minimally sufficient condition on any extension of the factor frame. Or put differently, a conjunction of factors is minimally sufficient for a given effect $E$ if, and only if, it contains only INUS conditions of $E$. Such a conjunction satisfies HP with respect to $E$, because it does not contain any irrelevant stuff. And so at least in this sense, the modern regularity theories imply HP.

With respect to VP, the answer is slightly more complex. Suppose a causal structure as the one illustrated by Fig. 4. It is based on the factor frame $\mathcal{F}_{0}:=\{A, B, C, D, E, F, G, H\}$ such that the factor set $A B C D$ is sufficient for $F G$, whilst no other factors that are located on the same level as $A, B, C$, and $D$ and that are not themselves causes of $A, B, C$, and $D$ are required to bring about $F G$. Suppose further that the higher-level factor $E$ supervenes on $A, B, C$, and $D$ (conjoined) and the higher-level factor $H$ supervenes on $F$ and $G$ (conjoined).

Under the assumptions that the factors in question are either "switched on" or "switched off", the structure of this kind will generate the data summarized in Table 1. Each row represents an observed coincidence of factors. Since the regularity theory can determine the direction of causation only if, for any given effect, there are at least two independent type-level causes, the table assumes the 
Table 1 Coincidence data generated by a causal structure involving overlapping events of different grains

\begin{tabular}{lllllllllllll}
\hline & $A$ & $B$ & $C$ & $D$ & $E$ & $F$ & $G$ & $H$ & $a c E$ & $a c F$ & $a c G$ & $a c H$ \\
\hline $\mathrm{c}_{1}$ & 1 & 1 & 1 & 1 & 1 & 1 & 1 & 1 & 0 & 0 & 0 & 0 \\
$\mathrm{c}_{2}$ & 0 & 1 & 1 & 1 & 0 & 0 & 1 & 0 & 0 & 0 & 0 & 0 \\
$\mathrm{c}_{3}$ & 1 & 0 & 1 & 1 & 0 & 0 & 1 & 0 & 0 & 0 & 0 & 0 \\
$\mathrm{c}_{4}$ & 1 & 1 & 0 & 1 & 0 & 1 & 0 & 0 & 0 & 0 & 0 & 0 \\
$\mathrm{c}_{5}$ & 1 & 1 & 1 & 0 & 0 & 1 & 0 & 0 & 0 & 0 & 0 & 0 \\
$\mathrm{c}_{6}$ & 0 & 0 & 1 & 1 & 0 & 0 & 1 & 0 & 0 & 0 & 0 & 0 \\
$\mathrm{c}_{7}$ & 0 & 1 & 0 & 1 & 0 & 0 & 0 & 0 & 0 & 0 & 0 & 0 \\
$\mathrm{c}_{8}$ & 0 & 1 & 1 & 0 & 0 & 0 & 0 & 0 & 0 & 0 & 0 & 0 \\
$\mathrm{c}_{9}$ & 1 & 0 & 0 & 1 & 0 & 0 & 0 & 0 & 0 & 0 & 0 & 0 \\
$\mathrm{c}_{10}$ & 1 & 0 & 1 & 0 & 0 & 0 & 0 & 0 & 0 & 0 & 0 & 0 \\
$\mathrm{c}_{11}$ & 1 & 1 & 0 & 0 & 0 & 1 & 0 & 0 & 0 & 0 & 0 & 0 \\
$\mathrm{c}_{12}$ & 1 & 0 & 0 & 0 & 0 & 0 & 0 & 0 & 0 & 0 & 0 & 0 \\
$\mathrm{c}_{13}$ & 0 & 1 & 0 & 0 & 0 & 0 & 0 & 0 & 0 & 0 & 0 & 0 \\
$\mathrm{c}_{14}$ & 0 & 0 & 1 & 0 & 0 & 0 & 0 & 0 & 0 & 0 & 0 & 0 \\
$\mathrm{c}_{15}$ & 0 & 0 & 0 & 1 & 0 & 0 & 0 & 0 & 0 & 0 & 0 & 0 \\
$\mathrm{c}_{16}$ & 0 & 0 & 0 & 0 & 0 & 0 & 0 & 0 & 0 & 0 & 0 & 0 \\
$\mathrm{c}_{17}$ & 0 & 0 & 0 & 0 & 1 & 0 & 0 & 1 & 1 & 0 & 0 & 0 \\
$\mathrm{c}_{18}$ & 0 & 0 & 0 & 0 & 0 & 1 & 0 & 0 & 0 & 1 & 0 & 0 \\
$\mathrm{c}_{19}$ & 0 & 0 & 0 & 0 & 0 & 0 & 1 & 0 & 0 & 0 & 1 & 0 \\
$\mathrm{c}_{20}$ & 0 & 0 & 0 & 0 & 0 & 0 & 0 & 1 & 0 & 0 & 0 & 1 \\
$\mathrm{c}_{21}$ & 0 & 0 & 0 & 0 & 0 & 1 & 1 & 1 & 1 & 1 & 0 & 0 \\
\hline
\end{tabular}

hypothetical existence of factors $a c E, a c F, a c G$ and $a c H$, which are presumed to be alternative causes of $F, G$, and $H$, respectively. ${ }^{13}$

Data of this kind poses a challenge to the modern regularity theories of causation in several ways. First, from the coincidence table it can be uncovered that the factor set $A B$ is minimally sufficient for $F$ (row $\mathrm{c}_{11}$ shows the sufficiency, whereas rows $\mathrm{c}_{2}-\mathrm{c}_{3}, \mathrm{c}_{6}-\mathrm{c}_{10}$, and $\mathrm{c}_{12}-\mathrm{c}_{16}$ prove the minimality). The existence of a second minimal sufficient condition for $F$-as indicated by row $\mathrm{c}_{18}$-allows us to interpret the relationships between $A$ and $F$ and $B$ and $F$ as causal (under certain further assumptions; cf. Baumgartner, 2008). Furthermore, factor set $C D$ is minimally sufficient for $G$, and the information contained in row $\mathrm{c}_{19}$ allows for a causal interpretation. Finally, $E$ is minimally sufficient for $H$, and by taking into account rows $\mathrm{c}_{20}$ and $\mathrm{c}_{21}$, this relationship is causally interpretable as well.

But now something strange happens. $A B C D$ turns out to be minimally sufficient for factor $E$, and the alternative cause $a c E$ of $E$ suggests that we have uncovered a causal relationship. Moreover, the factor set $F G$ is minimally sufficient for $H$, which by taking into account rows $\mathrm{c}_{17}$ and $\mathrm{c}_{20}$ is causally interpretable as well.

\footnotetext{
13 In order to avoid unnecessary complexity, the table does not make explicit all observable coincidences of factors $A-H, a c E, a c F, a c G$ and $a c H$, but includes only rows $\mathrm{c}_{17}-\mathrm{c}_{21}$. The omissions are indirectly indicated by the double line after row $\mathrm{c}_{16}$.
} 
Fig. 5 Proportionality for regularities

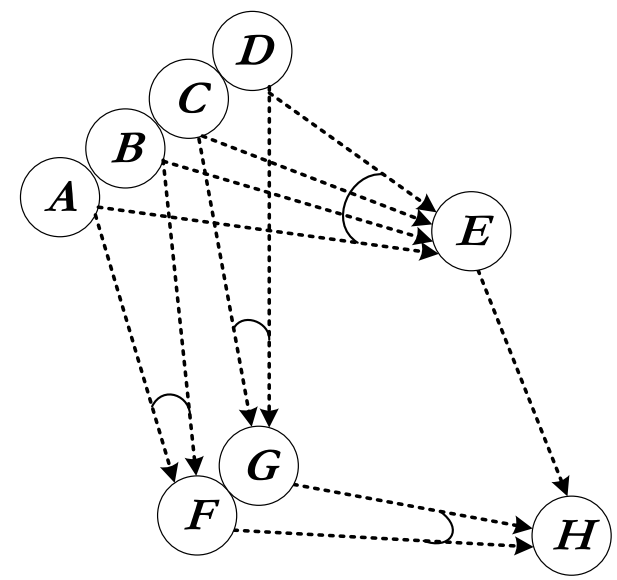

In short, based on concidence table data, the modern regularity theories cannot distinguish between a structure as the one illustrated by Fig. 4 and structures as the one illustrated by Fig. 5, which involves only causal relationships.

Even if additional information about the graininess of factors is introduced by declaring, perhaps, $\mathcal{F}_{1}:=\{A, B, C, D, F, G, a c F, a c G\}$ to be a fine-grained, or "lower-level", set and $\mathcal{F}_{2}:=\{E, H, a c E, a c H\}$ to be a coarse-grained, or "higherlevel", set, for the regularity theory the connection between, for instance, $F G$ and $H$ will still have to be interpreted as a causal relationship, and the regularity theory will accept inter-level causation. This result is perhaps even desirable. For instance, in the debate on supervenience Leuridan (2012) has argued that no meaningful distinction can be made between causation and supervenience, and so both should be considered as different versions of causation. Since a regularity framework interprets mechanistic constitution as an instance of supervenience (cf. Harbecke, 2014b), Leuridan's claim mighty apply in an analogous way.

In contrast, if, for independent metaphysical reasons, one would like to introduce a distinction between causation and supervenience, VP as a constraint on causation would yield the desired result. Based on $\mathcal{F}_{1}$ and $\mathcal{F}_{2}$, it would characterize the relationships $A B \rightarrow F, a c F \rightarrow F, C D \rightarrow G, a c G \rightarrow G, a c E \rightarrow E, E \rightarrow H$, and $a c H \rightarrow H$ as causal relationships, and it would declare $A B C D \rightarrow E$ and $F G \rightarrow H$ to be non-causal regularities. That is because VP would allow causal relationships only among members of the factor set $\mathcal{F}_{1}:=\{A, B, C, D, F, G, a c F, a c G\}$ and among members of the factor set $\mathcal{F}_{2}:=\{E, H, a c E, a c H\}$. All other sufficiency relationships it would (correctly) interpret as representing supervenience relationships within a supervenience hierarchy.

What this shows is that proportionality in the sense of VP plays no explicit role for the modern regularity theories of causation so far. However, it is consistent with these and can be introduced in order to make a distinction between causal and supervenience regularities for which the modern regularity theories of causation are blind otherwise. 
In conclusion then, the regularity theory of causation already in some sense implies HP through its minimal sufficiency. VP is not a topic explicitly dealt with in the literature on regularity theories. However, the core idea can be integrated into the theory and may even be a natural extension of it that solves a problem faced by them.

Are HP and VP to be interpreted ontically or epistemically in the context of regularity theories of causation? The answer depends on how one conceives of regularities. Hume himself may be read as having defended a non-theory, or antirealist theory of causation. "In reality, there is no part of matter, that does ever, by its sensible qualities, discover any power or energy, or give us ground to imagine, that it could produce any thing, or be followed by any other object, which we could denominate its effect." (1748, Sect. 7) In Hume's view, not even regularities are objective. Rather, they are perceived uniformities. Hence, if Hume would have accepted HP through minimization and VP as an additional constraint, he probably would have declared both to be purely epistemic principles.

Modern regularity theorists such as Mackie, May, Graßhoff, and Baumgartner are less explicitly empiricist and, in their rhetoric, seem to presuppose the objectivity of regularities. They provide both a definition of causation and principles for causal discovery (cf. Graßhoff \& May, 1995; Baumgartner and Graßhoff, 2004). The latter is, of course, compatible with Hume. Hence, at least within the modern frameworks, HP and VP could in principle be interpreted ontically within a regularity framework.

Concerning the modal version of Humean causal theory, it has already been mentioned in Sect. 2 that Yablo, (1992) grounds his theory of mental causation in a counterfactual theory of causation (cf. also Harbecke, 2014a). He introduces HP and VP as additional constraints yielding the result that mental events sometimes are causes. There have been some attempts to show that VP is actually implied by the counterfactual theory. As List and Menzies (2009) claim:

Assuming that the closest worlds in which the monkey does not have neural property $\mathrm{N} 1$ are ones in which it has another neural property realizing the intention I1, one can see that the second counterfactual is false: in any such world, the monkey has another neural property that realizes I1, and so performs A1." (488)

With this assumption in place, they can show that some scenarios involve higherlevel causation, namely when the higher-level events is "realization insensitive".

The problem is that, at least from the viewpoint of the classical counterfactual theory of causation, List and Menzies have overlooked a technical challenge by ignoring the "ban on replacement" (Bennett, 2003, fn. 21; cf. also Lewis, 2000, 190 and Lewis, 1986, 211). According to this principle, the possible world to be used for the evaluation of a counterfactual should not be one in which the event referred to by the antecedent of a counterfactual is replaced by another one. Rather, the event must be deleted and gone in that possible world, as though one applied a "metaphysical hole puncher". Otherwise, the counterfactual theory of causation will fail to identify any cause for (almost) any actual effect. If so, even 
if the higher-level event is realization insensitive, one does not get the result that the lower-level counterfactual is false.

If $\mathrm{M}$ supervenes on $\mathrm{N} 1$ without being "realization sensitive" (i.e. the closest non-N1 world is not a non-M world), then the closest non-N1 world relevant for the counterfactual interpretation is not one in which a replacer N2 is present. This observation shows that $\mathbf{V P}$ is not implied by the counterfactual theory. It would have to be added as an additional principle or constraint on counterfactual causation. This is the move that Yablo proposes in his paper.

Are HP and VP ontic or epistemic concepts under Yablo's definition? This again depends on the interpretation of the modalities involved. In Lewis' modal realism, causation and causal proportionality should probably be considered ontic relations. In Stalnaker's modal anti-realism (cf. Stalnaker, 1968), for instance, it is less clear that the relationship is an ontic one. In short, both options remain open, and they depend on further background assumptions about the ontology of possible worlds.

\section{Power Theories}

The power theory of causation is connected to Shoemaker's theory of properties as sets of powers. Shoemaker's central notion is that of the bestowing of powers through properties: "A property $\mathrm{P}$ bestows a causal power $\mathrm{C}(\mathrm{K}, \mathrm{E})$ upon the objects that have it just in case instances of $\mathrm{P}$, in circumstances $\mathrm{K}$, cause (or contribute to causing) instances of $\mathrm{E}$, and the holding of $\mathrm{K}$ alone does not cause (or contribute to causing) instances of E." (cf. Shoemaker, 1980).

The very notion of properties as "sets" suggests a special way in which properties can be related to one another. According to the author, “(...) sometimes the conditional powers bestowed by one property will be a proper subset of those bestowed by another." (Shoemaker, 2003b, 431) Since higher-level properties are just that-subsets of powers of fundamental properties - the subset relation makes clear, in Shoemaker's view, that higher-level properties do not compete with lower-level ones. As he says about the alleged competition of two properties P1 and P2, where P2 contains a subset of the powers of P1:

Yablo's notion of proportionality can be applied here. Where the only causal features of property P1 that play a role in producing an effect are ones that belong to property $\mathrm{P} 2$, of which $\mathrm{P} 1$ is a determinate or realizer property, there seems a good sense in which considerations of proportionality favor the instantiation of P2 over the instantiation of P1 as a cause of the effect. (Shoemaker, 2003b, 436)

In other words, Shoemaker considers a proportionality constraint to be not implied by, but compatible with, his power theory of causation. However, it is not entirely clear whether the kind of proportionality considered by Shoemaker corresponds to VP, HP, or both.

If the higher-order property is a subset of a lower-level property, it seems that a principle very similar to that of $\mathbf{H P}$ is intended. Shoemaker might have had in mind something like the following example. If property $P_{1}$ containing powers 
$\left\{p_{1}, p_{2}, p_{3}\right\}$ is a subset of property $P_{2}$ containing powers $\left\{p_{1}, p_{2}, p_{3}, p_{4}\right\}$, and for some effect property $P_{3}$ containing powers $\left\{p_{5}, p_{6}, p_{7}\right\}$, only powers $p_{1}, p_{2}$, and $p_{3}$ are required but not $p_{4}$, then $P_{1}$ is, and $P_{2}$ is not, proportional to $P_{3}$. In other words, the proportionality constraint as intended by Shoemaker may be isomorphic to the minimality condition of the regularity theory (cf. Sect. 5).

Whether VP has any application in this context is not obvious. That is because it is not entirely clear whether Shoemaker accepts that there are some non-overlapping properties or sets of powers that are connected by supervenience and are realized in the same place and time. If the answer to this ontological question is "yes", Shoemaker could introduce a version of proportionality in the sense of VP. However, again, his theory does not imply such a constraint.

It is also not clear whether Shoemaker intends proportionality as epistemic or ontic. Since powers in his view are objective features of the world, it seems natural to conclude that Shoemaker has an ontic view of proportionality as well. However, since his theory is in principle interpretable as a non-realism about causation, proportionality may also turn out as an epistemic concept in his overall framework. Both options seem open for the unconstrained power theory of causation.

\section{Conclusion}

This paper offered an investigation into the content of proportionality as a constraint on causation. It first reviewed some contributions in the philosophical literature that explicitly discuss, and in some cases adopt, Yablo's causal proportionality constraint. Subsequently, two general versions of the constraint were distinguished, namely "horizontal" and "vertical" proportionality. Moreover, the paper analyzed the relationship between the notion of proportionality and some of the classical theories of causation. With respect to each theory, it was discussed whether proportionality should be considered as an ontic, or as an epistemic, i.e. explanatory, constraint. Both positions can be found in the mental causation literature.

The following main claims were defended explicitly:

1. The horizontal (HP) and the vertical version (VP) of the proportionality constraint are logically independent; they do not entail each other or the other's negation.

2. HP is implied by some of the currently most popular theories of causation.

3. None of the currently most popular theories of causation contradicts either $\mathbf{H P}$ or VP. They could embrace HP and VP as further constraints, without rendering the underlying theory of causation incoherent.

4. HP and VP are not ontic or epistemic principles as such; rather, whether they are ontic or epistemic depends on the theories chosen plus background assumptions about the existence of higher-level causes and their non-identity to lower-level ones. 
Due to lack of space, a number of further questions concerning the proportionality constraint could not be discussed in detail in the present paper.

For instance, one further important class of theories of causation was not discussed, namely process theories as defended by Wesley Salmon (1984, 1997, 1998) and Phil Dowe (1992a, b). As it turns out, Dowe (2010) is quite sympathetic to the idea of proportionality as a constraint on causation. However, a deeper analysis of the role of proportionality in process theories of causation will have to await a future contribution on the topic.

Secondly, Sect. 3 only offered a preliminary motivation for the acceptance of proportionality as a constraint on causation. Much more would have to be said if a full defense of this substantial constraint should be considered reasonable.

Moreover, whether proportionality makes sense from a scientific, and not merely an colloquial, point of view could not be investigated in depth.

Finally, a formal proof of the logical independence of the two proportionality constraints was not offered, and the adaptions of HP and VP to the context of each particular theory of causation could be offered only in a preliminary way. Answering these questions and meeting these challenges will have to be left for future research on the topic of the proportionality constraint on causation.

Funding Open Access funding enabled and organized by Projekt DEAL. The fund was provided as Deutsche Forschungsgemeinschaft (No. HA6345/5-1). This work has been supported by the Deutsche Forschungsgemeinschaft (DFG), Reference Number HA 6349/5-1, Project Number 413568662; Project: "Model-Development in Neuroscience: Simplicity and Generalizability in Mechanistic Explanations".

Open Access This article is licensed under a Creative Commons Attribution 4.0 International License, which permits use, sharing, adaptation, distribution and reproduction in any medium or format, as long as you give appropriate credit to the original author(s) and the source, provide a link to the Creative Commons licence, and indicate if changes were made. The images or other third party material in this article are included in the article's Creative Commons licence, unless indicated otherwise in a credit line to the material. If material is not included in the article's Creative Commons licence and your intended use is not permitted by statutory regulation or exceeds the permitted use, you will need to obtain permission directly from the copyright holder. To view a copy of this licence, visit http://creativecommons.org/licen ses/by/4.0/.

\section{References}

Baker, A. (2016). Simplicity. In E. N. Zalta (Ed.), The Stanford encyclopedia of philosophy (Winter 2016 ed.). Metaphysics Research Lab, Stanford University.

Baumgartner, M. (2008). Regularity theories reassessed. Philosophia, 36(3), 327-354.

Baumgartner, M. (2009). Interventionist causal exclusion and non-reductive physicalism. International Studies in the Philosophy of Science, 23(2), 161-178.

Baumgartner, M. (2010). Interventionism and epiphenomenalism. Canadian Journal of Philosophy, 40(3), 359-383.

Baumgartner, M. (2013). Rendering interventionism and non-reductive physicalism compatible. Dialectica, 67(1), 1-27.

Baumgartner, M., \& Falk, C. (2019). Boolean difference-making: A modern regularity theory of causation. The British Journal for the Philosophy of Science., 5, 19. 
Baumgartner, M., \& Graßhoff, G. (2004). Kausalität und kausales Schliessen: eine Einführung mit interaktiven Übungen. Bern Studies in the History and Philosophy of Science.

Bennett, K. (2003). Why the exclusion problem seems intractable, and how, just maybe, to tract it. Noûs, 37(3), 471-497.

Bernstein, S. (2014). Two problems for proportionality about omissions. Dialectica, 68(3), 429-441.

Bontly, T. D. (2005). Proportionality, causation, and exclusion. Philosophia, 32(1), 331-348.

Brenner, A. (2017). Simplicity as a criterion of theory choice in metaphysics. Philosophical Studies, 174(11), 2687-2707.

Crane, T. (2008). Causation and determinable properties: On the efficacy of colour, shape, and size. See Hohwy and Kallestrup, 2008, 176-195.

Dowe, P. (1992). Process causality and asymmetry. Erkenntnis, 37(2), 179-196.

Dowe, P. (1992). Wesley Salmon's process theory of causality and the conserved quantity theory. Philosophy of Science, 59(2), 195-216.

Dowe, P. (2010). Proportionality and omissions. Analysis, 70(3), 446-451.

Gebharter, A. (2017). Causal exclusion and causal bayes nets. Philosophy and Phenomenological Research, 95(2), 353-375.

Gebharter, A., \& Eronen, M. (2021). Quantifying proportionality and the limits of higher-level causation and explanation. British Journal for the Philosophy of Science., 3, 1968.

Gillett, C., \& Rives, B. (2005). The non-existence of determinables: Or, a world of absolute determinates as default hypothesis. Noûs, 39(3), 483-504.

Graßhoff, G., \& May, M. (1995). Methodische analyse wissenschaftlichen entdeckens. Kognitionswissenschaft, 5, 51-67.

Graßhoff, G., \& May, M. (2001). Causal regularities. In W. Spohn, M. Ledwig, \& M. Esfeld (Eds.), Current issues in causation (pp. 85-114). Paderborn: Mentis.

Green, C. D., \& Vervaeke, J. (1996). What kind of explanation, if any, is a connectionist net. In C. W. Tolman, F. Cherry, R. van Hezewijk, \& I. Lubek (Eds.), Problems of theoretical psychology (pp. 201-208). North York: Captus.

Harbecke, J. (2008). Mental causation. Investigating the Mind's powers in a natural world. Frankfurt A.M.

Harbecke, J. (2013). On the distinction between cause-cause exclusion and cause-supervenience exclusion. Philosophical Papers, 42(2), 209-238.

Harbecke, J. (2014). Counterfactual causation and mental causation. Philosophia, 42(2), 363-385.

Harbecke, J. (2014). The role of supervenience and constitution in neuroscientific research. Synthese, 191(5), 725-743.

Harbecke, J., \& Atmanspacher, H. (2012). Horizontal and vertical determination of mental and neural states. Journal of Theoretical and Philosophical Psychology, 32(3), 161-179.

Hohwy, J., \& Kallestrup, J. (2008). Being reduced: New essays on reduction, explanation, and causation. New York: Oxford University Press.

Huemer, M. (2009). When is parsimony a virtue? The Philosophical Quarterly, 59(235), 216-236.

Hume, D. (2000/1748). An enquiry concerning human understanding: a critical edition. Oxford University Press, Oxford

Kim, J. (2003). Blocking causal drainage and other maintenance chores with mental causation. Philosophy and Phenomenological Research, 67(1), 151-176.

Kriegel, U. (2013). The epistemological challenge of revisionary metaphysics. Philosopher's Imprint, 13(12), 1-30.

Leuridan, B. (2012). Three problems for the mutual manipulability account of constitutive relevance in mechanisms. The British Journal for the Philosophy of Science, 63(2), 399-427.

Lewis, D. (1986). Postscripts to 'Causation.' In D. Lewis (Ed.), Philosophical papers (Vol. II, pp. 172213). New York: Oxford University Press.

Lewis, D. (2000). Causation as influence. The Journal of Philosophy, 97(4), 182-197.

List, C., \& Menzies, P. (2009). Non-reductive physicalism and the limits of the exclusion principle. Journal of Philosophy, 106, 475-502.

Mackie, J. (1974). The cement of the Universe. Oxford: Clarendon Press.

May, M. (1999). Kausales Schliessen. Eine Untersuchung über kausale Erklärungen und Theorienbildung. Ph.D. thesis, University of Hamburg, Germany.

McDonnell, N. (2017). Causal exclusion and the limits of proportionality. Philosophical Studies, 174(6), 1459-1474.

McGrath, M. (1998). Proportionality and mental causation: A fit? Nô̂s, 32, 167-176. 
McLaughlin, B. P. (2007). Mental causation and shoemaker-realization. Erkenntnis, 67(2), 149-172.

Pocheville, A., Griffiths, P. E., \& Stolz, K. (2017). Comparing causes: An information-theoretic approach to specificity, proportionality and stability. In H. Leitgeb, I. Niiniluoto, P. Seppälä, and E. Sober (Eds.), Proceedings of the Fifteenth International Congress of Logic, Methodology and Philosophy of Science, pp. 250-275. College Publications.

Raatikainen, P. (2010). Causation, exclusion, and the special sciences. Erkenntnis, 73(3), 349-363.

Salmon, W. (1984). Scientific explanation and the causal structure of the world. NJ: Princeton University Press Princeton.

Salmon, W. C. (1997). Causality and explanation: A reply to two critiques. Philosophy of Science, 49, 461-477.

Salmon, W. C. (1998). Causality: Production and propagation. In W. Salmon (Ed.), Causality and explanation (pp. 285-301). New York: Oxford University Press.

Schröder, J. (2007). Mental causation and the supervenience argument. Erkenntnis, 67(2), 221-237.

Schumacker, R., \& Lomax, R. (2004). A Beginner's guide to structural equation modeling. Lawrence Erlbaum.

Shapiro, L., \& Sober, E. (2007). Epiphenomenalism-the Do's and Don'ts. In G. Wolters \& P. Machamer (Eds.), Studies in causality: Historical and contemporary (pp. 235-264). University of Pittsburgh Press.

Shapiro, L. A. (2010). Lessons from causal exclusion1. Philosophy and Phenomenological Research, 81(3), 594-604.

Shoemaker, S. (2003). Identity, cause, and mind. Philosophical essays. Cambridge: Cambridge University Press.

Shoemaker, S. (2003b). Realization and mental causation. See Shoemaker (pp. 427-451).

Shoemaker, S. (2003/1980). Causality and properties. See Shoemaker (pp. 206-233).

Stalnaker, R. (1968). A theory of conditionals. Studies in Logical Theory, 2, 98-112.

Weslake, B. (2009). Exclusion excluded (unpublished). http://mail.rochester.edu/ bweslake/research/ papers/weslake_exclusion.pdf.

Weslake, B. (2013). Proportionality, contrast and explanation. Australasian Journal of Philosophy, 91(4), $785-797$.

Willard, M. B. (2014). Against simplicity. Philosophical Studies, 167(1), 165-181.

Woodward, J. (2003). Making things happen: A theory of causal explanation. New York: Oxford University Press.

Woodward, J. (2008a). Cause and explanation in psychiatry. In K. S. Kendler and J. Parnas (Eds.), Philosophical issues in psychiatry. Explanation, phenomenology, and nosology, pp. 132-184. Johns Hopkins University Press.

Woodward, J. (2008). Mental causation and neural mechanisms. See Hohwy and Kallestrup (pp. 218-262).

Woodward, J. (2015). Interventionism and causal exclusion. Philosophy and Phenomenological Research, 91(2), 303-347.

Woodward, J. (2016). The problem of variable choice. Synthese, 193(4), 1047-1072.

Woodward, J. (2021). Explanatory autonomy: The role of proportionality, stability, and conditional irrelevance. Synthese, 198(1), 237-265.

Yablo, S. (1992). Mental causation. The Philosophical Review, 101(2), 245-280.

Publisher's Note Springer Nature remains neutral with regard to jurisdictional claims in published maps and institutional affiliations. 